\title{
THE DETERMINATION OF ALL VARIETIES CONSISTING OF ABSOLUTELY CLOSED SEMIGROUPS
}

\author{
P. M. HIGGINS
}

\begin{abstract}
An example is given of a right normal band that is not absolutely closed. This enables us to determine all varieties of semigroups that consist entirely of absolutely closed semigroups.
\end{abstract}

1. Introduction Let $U, S$ be semigroups with $U \subseteq S$. Following Howie and Isbell [4] we say that $U$ dominates an element $d \in S$ if for every semigroup $T$ and all homomorphisms $\alpha, \beta: S \rightarrow T, \alpha|U=\beta| U$ implies $d \alpha=d \beta$. The set of all elements of $S$ dominated by $U$ is called the dominion of $U$ in $S$ and is denoted by $\operatorname{Dom}(U, S)$. It is easy to see that $\operatorname{Dom}(U, S)$ is a subsemigroup of $S$ containing $U$. We say that $U$ is closed in $S$ if $\operatorname{Dom}(U, S)=U$ and $U$ is absolutely closed if $U$ is closed in every containing semigroup $S$. We call a variety of semigroups $\mathcal{V}$ absolutely closed if $U \in \mathcal{V}$ implies $U$ is absolutely closed.

Semigroup dominions are characterised by the following celebrated theorem.

Result 1 (Isbell's Zigzag Theorem [5, Theorem 2.3]). Let $U$ be a subsemigroup of a semigroup $S$ and let $d \in S$. Then $d \in \operatorname{Dom}(U, S)$ if and only if $d \in U$ or there is a series of factorizations of $d$ as follows:

$$
d=u_{0} y_{1}=x_{1} u_{1} y_{1}=x_{1} u_{2} y_{2}=x_{2} u_{3} y_{2}=\cdots=x_{m} u_{2 m-1} y_{m}=x_{m} u_{2 m}
$$

where $m \geq 1, u_{i} \in U, x_{i}, y_{i} \in S$ with $u_{0}=x_{1} u_{1}, u_{2 i-1} y_{i}=u_{2 i} y_{i+1}, x_{i} u_{2 i}=$ $x_{i+1} u_{2 i+1}(1 \leq i \leq m-1)$ and $u_{2 m-1} y_{m}=u_{2 m}$.

Such a series of factorizations is called a zigzag in $S$ over $U$ with value $d$ and length $m$. In this paper we require just the "if" part of the statement of the zigzag theorem, a proof of which is an easy manipulation of the zigzag.

We use, whenever possible, and usually without comment, the notations and conventions of Clifford and Preston [1] or Howie [3].

2. The example. We give an example of a four-element right normal band which is not closed in a containing semigroup of seven elements.

Let $T_{6}$ denote the full transformation semigroup on $\{1,2,3,4,5,6\}$. We denote a particular member of $T_{6}$ by a list of the images of the domain elements $1,2, \ldots, 6$. Let $S$ be the subsemigroup of $T_{6}$ generated by the maps: $a_{1}=\left(\begin{array}{llll}1 & 1 & 1 & 444\end{array}\right), a_{2}=$ (113446), $a_{3}=(223556), x=(333666), y=(323656)$. Let $U$ be the subsemigroup of $S$ generated by $\left\{a_{1}, a_{2}, a_{3}\right\}$. The multiplication table for $S$ is given below.

Received by the editors January 11, 1982.

1980 Mathematics Subject Classification. Primary 20M07; Secondary 20M20. 


\begin{tabular}{c|ccccccc} 
& $a_{1}$ & $a_{1} a_{3}$ & $a_{2}$ & $a_{3}$ & $x$ & $y$ & $y a_{2}$ \\
\hline$a_{1}$ & $a_{1}$ & $a_{1} a_{3}$ & $a_{1}$ & $a_{1} a_{3}$ & $x$ & $x$ & $x$ \\
$a_{1} a_{3}$ & $a_{1}$ & $a_{1} a_{3}$ & $a_{1}$ & $a_{1} a_{3}$ & $x$ & $a_{1} a_{3}$ & $a_{1}$ \\
$a_{2}$ & $a_{1}$ & $a_{1} a_{3}$ & $a_{2}$ & $a_{3}$ & $x$ & $x$ & $x$ \\
$a_{3}$ & $a_{1}$ & $a_{1} a_{3}$ & $a_{2}$ & $a_{3}$ & $x$ & $a_{3}$ & $a_{2}$ \\
$x$ & $a_{1}$ & $a_{1} a_{3}$ & $x$ & $x$ & $x$ & $x$ & $x$ \\
$y$ & $a_{1}$ & $a_{1} a_{3}$ & $y a_{2}$ & $y$ & $x$ & $y$ & $y a_{2}$ \\
$y a_{2}$ & $a_{1}$ & $a_{1} a_{3}$ & $y a_{2}$ & $y$ & $x$ & $x$ & $x$
\end{tabular}

The semigroup $U=\left\{a_{1}, a_{2}, a_{3}, a_{1} a_{3}\right\}$ and is a right normal subband of $S$. Furthermore $x \in \operatorname{Dom}(U, S) \backslash U$ since the following is a zigzag in $S$ over $U$ with value $x$ :

$$
x=a_{1} y=x a_{1} y=x a_{2} y=x a_{3} y=x a_{3}
$$

and

$$
a_{1}=x a_{1}, a_{1} y=a_{2} y, x a_{2}=x a_{3}, a_{3} y=a_{3} .
$$

This example and its left-right dual show that the varieties of right normal bands and of left normal bands are not absolutely closed.

3. The absolutely closed varieties. All absolutely closed varieties of semigroups contain none of the following semigroups.

$A$, a three-element null semigroup,

$B$, a $2 \times 2$ rectangular band,

$C$, a two-element right zero semigroup with adjoined zero,

$D$, the right-left dual of $C$.

This is because $A$ is not absolutely closed [2] and nor is $B$ [4], while inspection of the lattice of bands [3, p. 124] shows that $C$ (respectively $D$ ) generates the variety of right (respectively left) normal bands.

THEOREM 2. The absolutely closed varieties of semigroups are exactly the varieties consisting entirely of semilattices of groups, or entirely of right groups or entirely of left groups.

REMARK. These varieties are the subvarieties of those defined by identities of the form: $\left[x=x^{1+n}, x^{n} y^{n}=y^{n} x^{n}\right.$, some $\left.n \geq 1\right]$ (semilattices of groups), $\left[x=x^{1+n}\right.$, $x^{n} y=y$ for some $n \geq 1$ ] (right groups) and $\left[x=x^{1+n}, y x^{n}=y\right.$ for some $\left.n \geq 1\right]$ (left groups).

PROOF OF THEOREM. The varieties listed in the statement of the theorem consist entirely of inverse semigroups, of right simple semigroups and of left simple semigroups respectively. These three types of semigroups are absolutely closed [4]. Hence the listed varieties are absolutely closed.

Conversely suppose $\mathcal{V}$ is an absolutely closed variety of semigroups. The threeelement null semigroup $A$ satisfies all nontrivial identities except those for which one side consists of a single variable $x$. Any such identity implies an identity of the kind $x=x^{1+n}$ for some $n \geq 1$. Since $A$ is excluded from $\mathcal{V}, \mathcal{V}$ therefore admits 
an identity of the form $x=x^{1+n}$ for some $n \geq 1$. Hence each $S \in \mathcal{V}$ is a union of groups and so is a semilattice of completely simple semigroups [3, Chapter 4, Theorem 1.7].

Let $D$ be any one of the completely simple semigroups occurring as a $D$-class in $S$. If $D$ is neither right simple nor left simple then $D / \not H$ (where $\not$ is Green's $\not$ relation on $D$ ) is a rectangular band which contains a $2 \times 2$ rectangular subband; this implies $B \in \mathcal{V}$, a contradiction. Therefore $D$ is a right group or a left group. However, if $\mathcal{V}$ contained a right group and a left group, both of which were not groups, then $\mathcal{V}$ would contain $R$ and $L$, a two-element right and a two-element left zero semigroup. This would imply that $B \simeq R \times L \in \mathcal{V}$, a contradiction. Hence $\mathcal{V}$ consists entirely of semilattices of right groups or entirely of semilattices of left groups.

Suppose $\mathcal{V}$ consists entirely of semilattices of right groups. Then either $\mathcal{V}$ consists entirely of semilattices of groups, entirely of right groups or $\mathcal{V}$ contains a nontrivial semilattice and a right group which is not a group. In the latter case $\mathcal{V}$ contains a two-element semilattice $T=\{0, e\}$ and a two-element right zero semigroup $R=$ $\{f, g\}$. The product $R \times T$ has an ideal $I=\{(f, 0),(g, 0)\}$. The factor semigroup $(R \times T) / I \simeq C$, implying $C \in \mathcal{V}$, a contradiction. Hence this alternative does not arise and so $\mathcal{V}$ consists entirely of semilattices of groups or entirely of right groups. The dual argument shows that if $\mathcal{V}$ consists entirely of semilattices of left groups then in fact $\mathcal{V}$ consists entirely of semilattices of groups or entirely of left groups. This completes the proof of the theorem.

I thank my supervisor, Tom Hall, for his guidance throughout the preparation of this paper. I give special thanks for his help together with that of Graham Clarke for their suggestions in proving Theorem 2.

\section{REFERENCES}

1. A. H. Clifford and G. B. Preston, The algebraic theory of semigroups, Math. Surveys, No. 7, Amer. Math. Soc., Providence, R.I., Vol. I, 1961; Vol. II, 1967.

2. T. E. Hall, Epimorphisms and dominions, Semigroup Forum 24 (1982), 271-283.

3. J. M. Howie, An introduction to semigroup theory, London Math. Soc. Monographs 7, Academic Press, New York, 1976.

4. J. M. Howie and J. R. Isbell, Epimorphisms and dominions. II, J. Algebra 6 (1967), 7-21.

5. J. R. Isbell, Epimorphisms and dominions, Proc. Conf. Categorical Algebra (La Jolla, 1965), Lange \& Springer, Berlin, 1966, 232-246.

Department of Mathematics, Monash University, Clayton, Victoria 3168, Australia 